evidence suggesting or demonstrating harm has grown'. He concludes that 'mounting awareness of the probable futility of antipsychotic polypharmacy is reflected in the latest guidance issued by the National Institute for Health and Clinical Excellence'.

Lepping \& Harbone ${ }^{2}$ draw attention to the need for a 'more balanced view with regard to polypharmacy in a patient group that is often non-responsive'. We would like to address issues raised by Odelola \& Ranceva. ${ }^{3}$

First, Odelola \& Ranceva speculate that the persistence of antipsychotic polypharmacy despite repeated guidance against it may indicate that this is one area where clinical practice is ahead of research evidence. They reiterate Lepping \& Harbone's point that in the case of polypharmacy the evidence provides no support either way - hardly a ringing endorsement. Additionally, they praise the excellent recommendations by Langan \& Shajahan. ${ }^{4}$ In the context of their letter we would be concerned that this is potentially misleading. Langan \& Shajahan urge extreme caution if one uses polypharmacy, supported by thorough explanatory documentation, rigorous monitoring and ongoing review. They conclude with the caveat that the 'worrying relationship' between the use of polypharmacy and mortality merits investigation and that it remains 'more art than science'. The message to take home seems to be 'avoid if possible'.

Among the routes to antipsychotic polypharmacy, nearly all of the researchers quoted here identify the failure to complete a switch from one agent to the other as a starting point for polypharmacy - this surely represents an opportunity for psychiatrists to tackle unplanned and inappropriate polypharmacy. The risks of high-dose prescribing should also be borne in mind.

The fact that there are probably increasing rates of polypharmacy prescribing should not be misinterpreted as evidence in support of it - once it was doubted by many that the world was spherical! Evidence suggests that the two polypharmacy scenarios outlined in the National Institute for Health and Clinical Excellence guidelines, ${ }^{5}$ cross-tapering and adding an antipsychotic to clozapine, appear reasonable. Outside these scenarios the risks $v$. benefits demand serious concern. We would echo Odelola \& Ranceva's call to be openminded about polypharmacy. This would extend to entertaining the possibility that the practice should be jettisoned in many cases. To cope with any overwhelming feelings of therapeutic nihilism, we would direct readers to Williams et al's editorial. ${ }^{6}$

1 Taylor D. Antipsychotic polypharmacy - confusion reigns. Psychiatrist 2010; 34: 41-3.

2 Lepping P, Harbone GC. Polypharmacy: how bad are we really? Psychiatrist 2010; 34: 208-9.

3 Odelola D, Ranceva N. Polypharmacy: saint or sinner? Psychiatrist 2010; 34: 354

4 Langan J, Shajahan P. Antipsychotic polypharmacy: review of mechanisms, mortality and management. Psychiatrist 2010; 34: 58-62.

5 National Institute for Health and Clinical Excellence. Schizophrenia: Core Interventions in the Treatment and Management of Schizophrenia in Primary and Secondary Care (Update). NICE, 2009.

6 Williams L, Newton G, Roberts K, Finlayson S, Brabbins C. Clozapineresistant schizophrenia: a positive approach. Br J Psychiatry 2002; 181 $184-7$

Rajesh Rajpal Regional North East GP Training Scheme, Elizabeth Owens senior registrar, National Higher Training Scheme, MacDara McCauley consultant psychiatrist, St Brigid's Hospital, Ardee, Co. Louth, Ireland, email: macdara.mccauley@hse.ie

doi: 10.1192/pb.35.1.31a

\section{Patient satisfaction rating scales $v$. patient-related outcome and experience measures}

We were pleased to read the paper by Hansen et al detailing their validation of a patient satisfaction rating scale. This sort of work is very much in keeping with the recent government paper, Equity and Excellence: Liberating the NHS. ${ }^{2}$ However, we thought that the focus of the questionnaire was too narrow: it essentially only dealt with the interaction between psychiatrist and service user in an out-patient setting. A far broader perspective would need to be taken for this instrument to be used as a service satisfaction questionnaire, because patients interact with a far greater range of people and systems as they move through a given care pathway. Even in a fairly circumscribed setting such as out-patient setting service users deal with appointment letters, receptionists, the physical environment of the waiting room, etc., even before they get to meet a psychiatrist. However, we did think that the questionnaire would make an excellent instrument for psychiatrists (and other mental health professionals) to use as part of their annual appraisal or multisource feedback, as it provides good information about the vital interaction between doctor and patient.

More pertinent to today's clinical practice are Patient Reported Outcome Measures (PROMs) and Patient Reported Experience Measures (PREMs). They provide richer information than patient satisfaction questionnaires, which are concerned with a relatively narrow (but obviously very important) area. It is possible for a user to have a satisfactory experience of a service (and score a satisfaction questionnaire highly) but a poor clinical outcome (which would not be identified by a satisfaction scale). On the other hand, PROMs and PREMs will capture not only the patient experience/ satisfaction but also the outcome from the patient's perspective. These data complement the gathering of routine clinical outcome data, which in the UK pertain primarily to the Health of the Nation Outcome Scales. ${ }^{3}$

Patient Reported Outcome Measures and Patient Reported Experience Measures have been established in acute trusts for quite so time now. They suit certain specialties well, for example post-hip operation PROMs are ubiquitous, but in mental health they are much rarer. To attempt to address this gap, we (and other colleagues from our trust) are in the process of validating a PROM/PREM for mental health service users. ${ }^{4}$ A version of the instrument specifically for use with older patients has already been successfully piloted and preliminary results will shortly be published. ${ }^{5}$

We would like to congratulate Hansen et al on their work. However, we believe that outcome data supersede patient satisfaction questionnaires in contemporary National Health Service practice. The latter can easily be incorporated into the PROMs/PREMs, which additionally provide a wider range of information.

1 Hansen LK, Vincent S, Harris S, David E, Surafudheen S, Kingdon D. A patient satisfaction rating scale for psychiatric service users. Psychiatrist 2010; 34: 485-8. 
2 Department of Health. Equity and Excellence: Liberating the NHS. TSO (The Stationery Office), 2010.

3 Wing JK, Beevor AS, Curtis RH, Park SB, Hadden S, Burns A. Health of the Nation Outcome Scales (HoNOS). Research and development. Br J Psychiatry 1998; 172: 11-8.

4 Whelan $\mathrm{P}$, Andrews T, Patel S, Lewis A. Pinning down opinion in mental healthcare. Health Serv J 2010; 4 Nov: 20-1.

5 Whelan $\mathrm{P}$, Lewis A, Patel S, Andrews T. Taking the Cinderella specialty to the PROM: developing a Patient-Related Outcome Measure for an older adult mental health service. Qual Ageing Older Adults. In press.

Paul J. Whelan is consultant old age psychiatrist, email: paul.whelan@ nhs.net, Leena Reddy is specialty doctor and Tresa Andrews is consultant clinical psychologist and Head of Psychology for Westminster, Central and North West London NHS Foundation Trust, North Westminster CMHT for Older Adults.

doi: 10.1192/pb.35.1.32

\section{Domestic violence is most commonly reciprocal}

Morgan et al highlight the high incidence of being a victim of intimate partner violence among female psychiatric patients in the UK. This is in keeping with a historic approach that has conceptualised domestic violence as something that men do to women and has only sought evidence for violence by men against women.

Partly this may be because women are more likely to report intimate partner violence than men. One study found that in the same sample of couples $28 \%$ of the women, but only $19 \%$ of their male partners, reported that their relationships were violent, suggesting underreporting in a third of men. ${ }^{2}$

In recent years researchers have approached populations without preconceptions as to the direction of violence. Large epidemiological studies have demonstrated that domestic violence is most commonly reciprocal and that when only one partner is violent there is an excess of violent women. Whitaker et $a_{1}{ }^{2}$ in a study of 14000 young US couples aged $18-28$ years, found that $24 \%$ of relationships had some violence and half of those were reciprocally violent. In $70 \%$ of the non-reciprocally violent relationships women were the perpetrators of violence. Reciprocal violence appears to be particularly dangerous, leading to the highest rate of injury (31.4\%). This may be because reciprocal violence is more likely to escalate.

The International Dating Violence Study ${ }^{3}$ found that among students at 31 universities worldwide male and female students had similar rates of physically assaulting a partner ( $25 \%$ of men and $28 \%$ of women at the median university). There was parity for perpetrating severe assaults (used a knife or gun, punched or hit partner with something that could hurt, choked partner, slammed partner against a wall, beat up partner, burned or scalded partner on purpose, kicked partner) - $9 \%$ of male and female students at the median university. For severe injury (passed out, required medical attention or broke a bone) the perpetration rate was higher for males (median rate $3.1 \%$ by men and $1.2 \%$ by women).

A review of 62 empirical studies of female-perpetrated intimate partner violence ${ }^{4}$ found rates of physical violence of 4-79\% among adolescent girls, $12-39 \%$ among female college students and $13-68 \%$ among adult women. The researchers concluded that a significant proportion of females seeking help for victimisation are also perpetrators of intimate partner violence, and that those who treat battered women may need to consider addressing the perpetration of violence with their female clients.

Archer ${ }^{5}$ attempted to resolve two competing hypotheses about partner violence, either that it involves a considerable degree of mutual combat or that it generally involves male perpetrators and female victims. His meta-analysis of 82 studies of gender differences in physical aggression between heterosexual partners showed that men were more likely to inflict an injury; $62 \%$ of those injured by a partner were women, but men still accounted for a substantial minority of those injured. However, women were slightly more likely than men to use one or more act of physical aggression and to use such acts more frequently. Younger aged couples showed more female-perpetrated aggression.

Only examining rates of violence perpetrated against women risks perpetuating an inaccurate stereotype of women as victims and men as aggressors. This may hinder women from receiving support to reduce their own perpetration of violence and may contribute to the underreporting of violence perpetrated by women against men.

1 Morgan JF, Zolese G, McNulty J, Gebhardt S. Domestic violence among female psychiatric patients: cross-sectional survey. Psychiatrist 2010; 34: 461-4.

2 Whitaker DJ, Haileyesus T, Swahn M, Saltzman LS. Differences in frequency of violence and reported injury between relationships with reciprocal and nonreciprocal intimate partner violence. Am J Public Health 2007: 97: 941-7.

3 Strauss $M$. Prevalence of violence against dating partners by male and female university students worldwide. Violence Against Women 2001; 10: $790-811$.

4 Williams JR, Ghandour RM, Kub JE. Female perpetration of violence in heterosexual intimate relationships. Trauma Violence Abuse 2008: 9; 227-49.

5 Archer J. Sex differences in aggression between heterosexual partners. A meta-analytic review. Psychol Bull 2000; 126: 651-80.

Daniel McQueen is consultant child and adolescent psychiatrist, Barnet Enfield and Haringey Mental Health Trust, London, email: Daniel.McQueen@barnet.nhs.uk

doi: $10.1192 / p b .35 .1 .33$

\section{What is the object of the psychiatrist's expertise?}

Craddock et $a l^{\prime}$ are to be congratulated for asking 'What is the core expertise of the psychiatrist?'. In responding to this rhetorical question, they make reference to psychological and social factors in mental illness; yet the impression remains that they consider biomedical factors central to psychiatry and the others more peripheral. Why else, for example, do they refer to diagnosis but not case formulation in psychiatry?

Craddock et al attempt to identify the expertise of the psychiatrist without first defining the object of his or her expertise. If the nervous system is the object of the neurologist's expertise and the whole person/family is the object of the general practitioner's expertise, what is the object of the psychiatrist's expertise? For lkkos et al ${ }^{2}$ this is affect. Affect refers to feelings, agitations and moods, which are manifested in consciousness, behaviour and relationships in family and society. It is disturbed affect that brings individuals to the attention of psychiatrists, whether voluntarily or not, especially when it cannot be contained in the family and primary care. Disturbed affect may be caused by neurological 